INTERNATIONAL DESIGN CONFERENCE - DESIGN 2018

https://doi.org/10.21278/idc.2018.0306

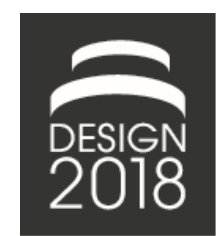

\title{
BALL BEARINGS AS SENSORS FOR SYSTEMATICAL COMBINATION OF LOAD AND FAILURE MONITORING
}

\author{
T. Schirra, G. Martin, S. Vogel and E. Kirchner
}

\begin{abstract}
In modern machine architecture, complex mechanical components guarantee the function of the system. Specific maintenance rates reduce maintenance effort and cost. The combination of load and failure monitoring provide data to establish an individual maintenance rate depending on component specific remaining lifetime. In addition, algorithms can optimise the operation strategy to relieve the weakest components and extend the system lifetime. Adapted ball bearings contain a sensor mechanism depending on the relation of beating load and electric bearing impedance to measure load and failure data.
\end{abstract}

Keywords: cyber physical systems, self optimising systems, smart products engineering, bearing impedance, load sensor

\section{Introduction: Monitoring reduces operating costs}

Maintenance and unexpected downtimes are key contributors of machine operating cost. By knowing the likely occurrence of a machine failure in advance, the operator can avoid unexpected downtime and resulting cost. Furthermore, delayed machine error leads to lowered maintenance rate and again reduced cost. Failure monitoring detects and load monitoring estimates the occurrence of machine failures. The proximity of bearings to the mechanical process leads to a high suitability of ball bearings for serve in addition as load condition sensors and thus failure monitors. Failure monitoring combines fault diagnosis and failure prognosis, both phenomena occur on machine components. The task of failure monitoring is to detect, to allocate and to quantify the state of the fault, thus resulting in an increased knowledge of mode and severity of a fault. Analysing the monitoring data leads to the Remaining Useful Life (RUL) of components (Mikat, 2015).

Failure Prognosis is an estimation of the future trend up to a component failure based fault status and estimated loads (Mikat, 2015). Failure monitoring thus enables condition based predictive maintenance. The knowledge of the RUL is crucial for the exploiting of the potential of dynamic maintenance. In addition to failure monitoring, systems can adopt load monitoring in parallel. While failure monitoring detects an incipient crack, load monitoring determines the overall load configuration of components in loaded state (Foulard, 2015).

Lifetime estimations as a part of the designing process depend on knowledge about loads and load capacity of the component. The more accurate the knowledge of the loads and load capacity, the more accurate is the estimation of the lifetime. Therefore, usage of load monitoring improves the accuracy of lifetime estimation caused by reducing the uncertainty in load assumptions (Foulard, 2015). The following method combines benefits of load and failure monitoring.

Failure monitoring achieves the possibility of an accurate RUL estimation for components of systems, which have an incipient crack. For all the other components of the system, the estimation of lifetime is 
possible by means of load monitoring. Estimations based on measured load data are less accurate than estimations by failure data because of the uncertainty in the load capacity, but more accurate than estimation without load monitoring because of the reduction of uncertainty in loads. The combination of load and failure monitoring can extend the maintenance rate and fully utilise the useful life of components. The components remain in operation until the end of their lifetime. The presented sensor mechanism detects the loads on ball bearing by measuring the electric impedance (Prashad, 2006). With few modifications, mechanical systems can use ball bearings for load monitoring. This offers the possibility to combine load and failure monitoring in a practical and wide spreading way.

\section{Example: Switchable transmission}

Figure 1 illustrates a schematic view of a switchable two-speed transmission. Engine 1 drives shaft 2 with two pivot mounted pinions 4 and 5. Bearings 9 and 10 carry the shaft in the housing 13 . The shift collar 8 switches pinion 4 or 5 into the flowlines of force. Shaft 3 carries the driven gears 6 and 7 , bearing 11 and 12 support the output shaft.

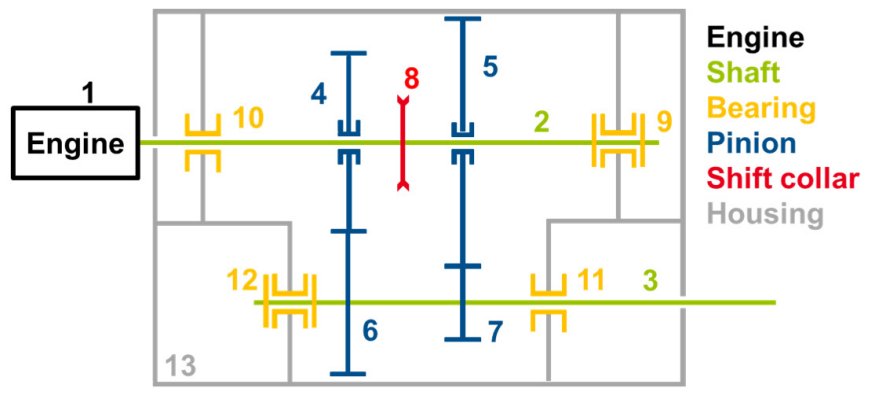

Figure 1. Schematic view of a transmission with two switchable gears

In order to enable suitable combination of load and failure monitoring, component specific load and failure data by different sensors is mandatory. Therefore sensors have to measure loads and fault of every single component used in the transmission. In the example given, the sensors mounted in shift collar 8 and bearings 10 and 12 generate a net of sensors for backtracking loads and faults of all components. The sensor mechanism for ball bearings, presented in this paper to support the structure of a spread sensor net in this transmission. All components are dependent on each other; Figure 2 illustrates a simplification of the mechanical dependencies of the transmission's components. The schematic view neglects other effects, e.g. thermal effects. Engine 1 delivers torque and rotation speed to shaft 2. Combining the data of the machine elements with sensors leads to component specific load and failure data. The red framed sensor fitted bearings 10 and 11 and the yellow framed sensor fitted shift collar generate direct data of the surrounding components. Only components 6 and 7 are not in direct contact to a sensor fitted component. The consequences are models to backtrack the monitoring data.

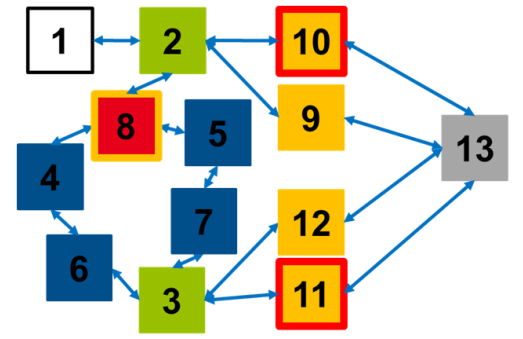

Figure 2. Mechanical dependence in transmission of Figure 1 - sensor fitted components are framed

During the design process, engineers need to compare loads and load capacity to estimate their expectable lifetime equal to the requirements. A failure occurs if overall load configuration exceeds the load capacity. Loads and load capacity contains uncertainty because of inaccuracy in the estimation of the load applied on a component during its lifetime, the variation of material properties and variation in the 
manufacturing process of the component itself.. This results in a range of load and load capacity of every single component, Figure 3. The resulting range of lifetime estimation can be more accurate, by reducing the previously mentioned uncertainty. Uncertainty consists of different types. Model-based uncertainty leads to uncertainty in the overall load configuration. Loads are predicted/determined by models that approximate the reality. This leads to a difference between model-based loads and real applied load, called model-based (Würtenberger, 2017). Uncertainty in the production process leading to uncertainty in load capacity. Manufacturing tolerances can occur in the production of the wrought material or other machining process and cause uncertainty. The combination of load and failure Monitoring can reduce uncertainty in load and load capacity and therefore improve the accuracy of lifetime estimation.

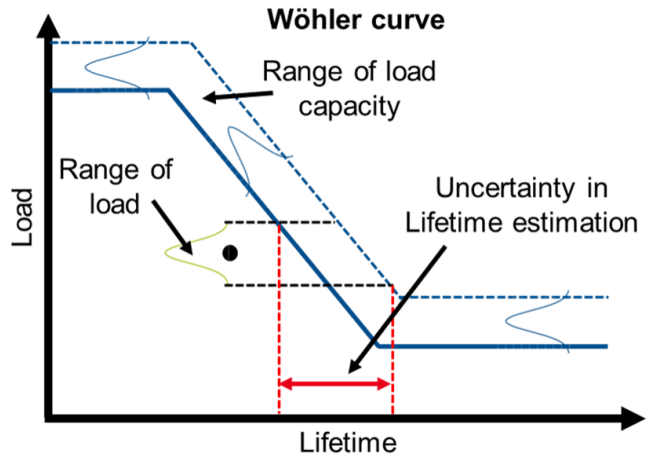

Figure 3. Effect of uncertainty in load and load capacity on lifetime estimation

\section{Combination of load and failure monitoring}

The combination of load and failure monitoring combines the advantages of both approaches to extend the maintenance rate and fully utilises the component lifetime. The method consists of three stages of a circle. Regarding Figure 4, the different stages build one another beginning with the monitoring. The first stage enables a parallel load and failure monitoring for every single system's component. Monitoring result is a component specific estimated lifetime, based on the measured overall load configuration. Calculations based on estimated lifetime and the overall load configuration result in component specific estimated RUL. Simultaneous failure monitoring in stage I tries to detect an incipient crack at one of the system's components. Based on detected incipient crack, the component specific RUL estimation begins depending on the future loads. The second stage is a comparison of the component's estimated RUL and the RUL of the component with the incipient crack. The results of stage II are the boundary conditions for the optimisation of the operating conditions in stage III. The load on components with a less RUL shall be reduced, while components with a higher RUL experience an increased load. The goal is to distribute the loads based on the results of stage II to balance the lifetime of the components. The method is an iterative process. Repetitions improve the results by virtue of an improved knowledge and therefore reducing uncertainty in loads and load capacity. The result is a lower rate of maintenance and a higher utilisation factor.

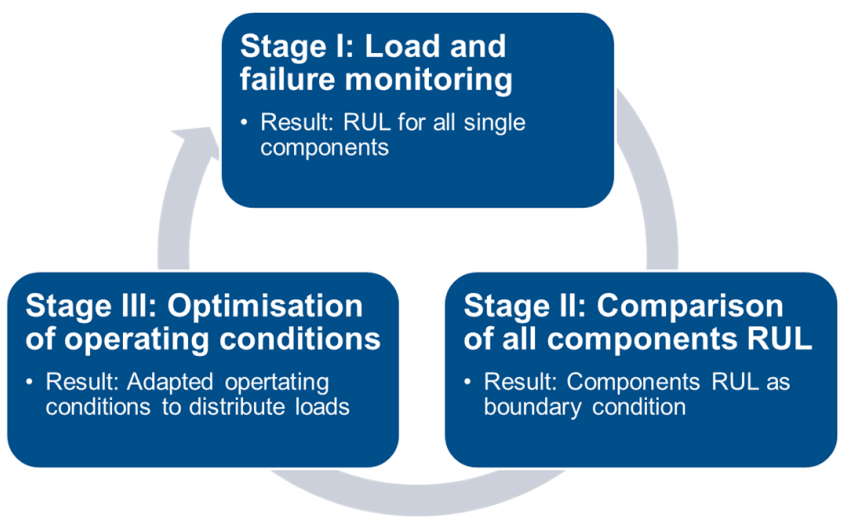

Figure 4. Combination of load and failure monitoring 


\section{Stage I}

Load monitoring means measuring the overall load configuration in operating conditions. The goal is getting to know specific load conditions for every single component and minimise uncertainty in load assumptions. The minimised uncertainty causes a minimal range of possible load leading to a more accurate lifetime estimation, comparing Figure 3 and Figure 5a. The estimated RUL for all components with Load Monitoring depends on worst-case estimated lifetime, overall load configuration and future loads.

Failure monitoring detects an incipient crack of one component of the system. Depending on the fault and the future loads, there is an RUL for component, compare to Figure 5b.
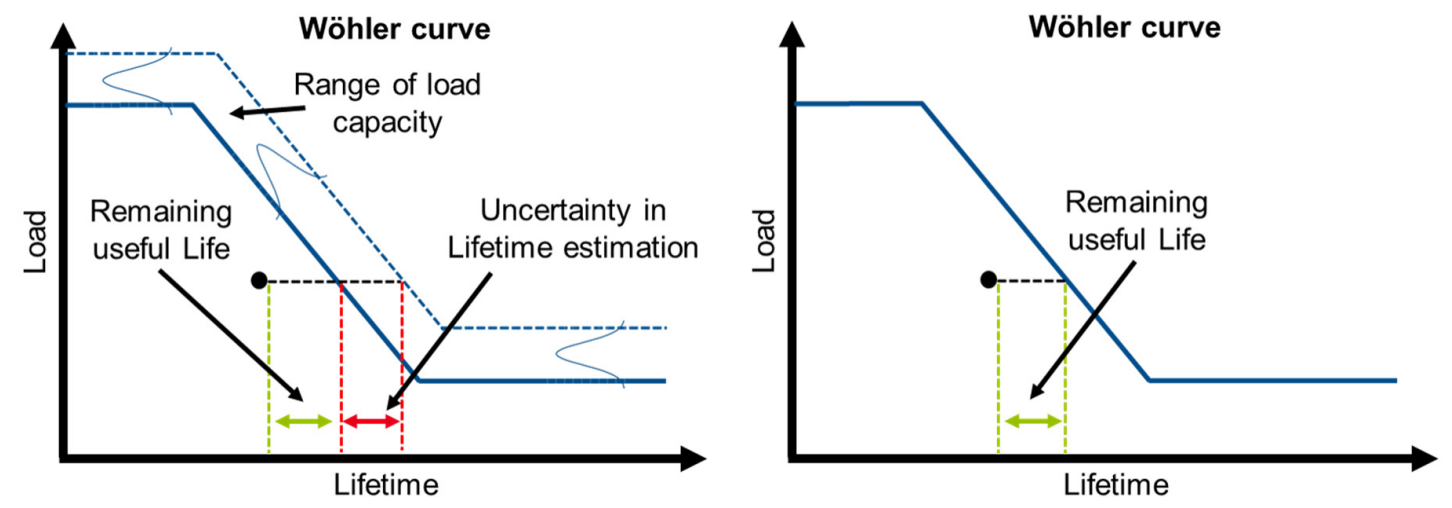

Figure 5. Left: Result of load monitoring (a); right: Result of failure monitoring (b)

\section{Stage II}

The Comparison of the different RUL for every single component with and without incipient cracks is the goal of stage II. For example, the presented transmission has an incipient crack at pinion 5 detected by failure monitoring with the sensor net. Pinion 5 has RUL with the value X. The result of the load monitoring of pinion 4 is a RUL with the value Y. Figure 6 shows a cut-out of the results of stage II, comparing the RUL of pinion 4 and 5 with the RULs as boundary condition for stage III.

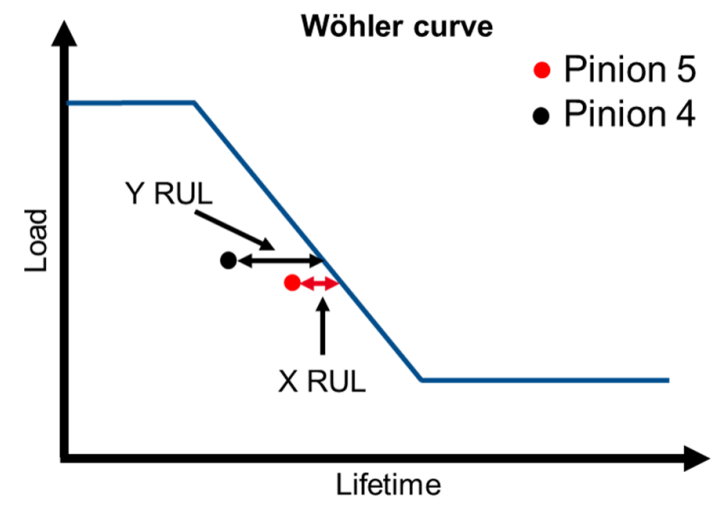

Figure 6. Comparison RUL of pinion 4 and 5

\section{Stage III}

The last stage consist of optimising the remaining lifetime. The best-case scenario stage is an equal remaining lifetime for every single component. This is challenging to achieve because of the uncertainty in load capacity of the explicit components, which influences the RUL. Adapting the system's operating conditions leads to adapted future loads in order to achieve a similar remaining lifetime for components with different values of RUL as boundary condition. The optimisation process translocates the loads, from the components with low value of RUL to components with high value of RUL, in order to reach an equal remaining lifetime. The transmission's operating strategy reduces load of pinion 5 and increases load of pinion 4 . The extended usage of pinion 4 represents a part of the optimised operating condition, especially in cases of load peaks. In addition, the operating strategy limits the engine output if pinion 5 
remains in the flowline of force. By this remaining lifetime of the component with the incipient crack extends. The maintenance rate lowers prolong. In addition, components, changed anyway at next maintenance service, are more utilised with the help of combined load and failure monitoring. Both aspects can reduce operating costs.

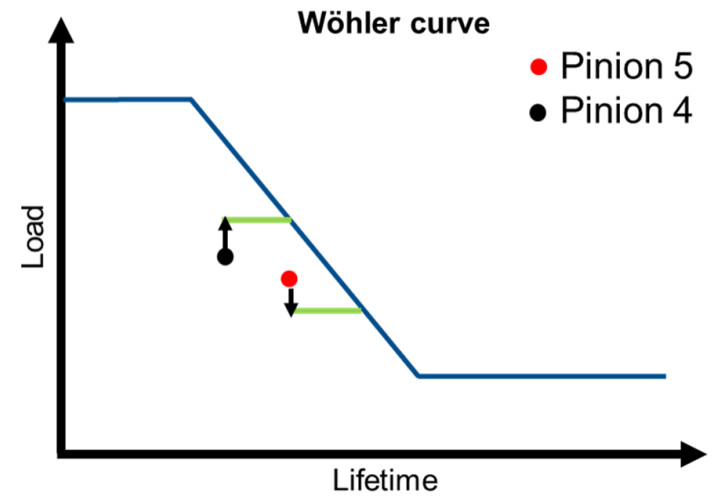

Figure 7. Remaining lifetime of pinion 4 and 5 with optimised operating conditions

The implementation of this method in practice allows individual maintenance at the required time. The lifetime of the weakest component with the incipient crack limits the rate of maintenance. This component's lifetime is an essential part of the optimisation. Reaching the estimated lifetime of one bearing usually leads to a change of all bearings. For example when bearing 9 of the transmission reached the end of lifetime, there is also a change of bearings 10,11 and 12 even if they are far away from their estimated lifetime, because the transmission is disassembled anyway and the change is costefficient. By monitoring failure and load, the other bearings are much closer to their end of lifetime when bearing 9 reaches the end of its lifetime.

\section{Use of ball bearing as sensors}

Implementing the combination of load and fault monitoring into practice requires significant load and failure data for each component. While failure monitoring focusses on system vibrations as failure data (Mikat, 2015), there is a need of information about forces and torque at every single component as load data. One efficient possibility is to measure load and failure data with one sensor. A sensor position to measure both data is to fit sensors into force transmitting machine elements (Martin et al., 2018). If the frequency of load changes is different to the frequency of vibrations caused by incipient cracks, those machine elements can differ loads and faults. If there is a sufficient amount of sensors placed in machine elements of a system, there is a net of sensors to monitor loads and failures on every single component. Bearings are machine elements, which are suitable to fit a sensor. Many rotating systems use ball bearings and forces on a rotating shaft always cause forces to bearings. They are a wide spread type in technical systems.

\subsection{Impedance model of ball bearings}

The electric impedance of ball bearings depends on the hertzian area between raceway and balls and the lubrication thickness (Prashad, 2006). Beside geometry and material parameter, the hertzian area depends only on bearing forces while the lubrication thickness depends on operating conditions as temperature, speed and bearing forces. The relation of operating conditions to hertzian area and lubrication thickness is already researched and can be estimated (Radnai et al., 2015). The estimation of the bearing forces predicated on measuring temperature, speed and electric impedance. The model of calculating bearing impedance consists of the electric analogous model of all rolling contacts (Gemeinder, 2016). Different lubrication conditions lead to different electric properties of rolling contacts and therefore three different electric analogous models. Figure 8 shows the different lubrication conditions of rolling contacts. 


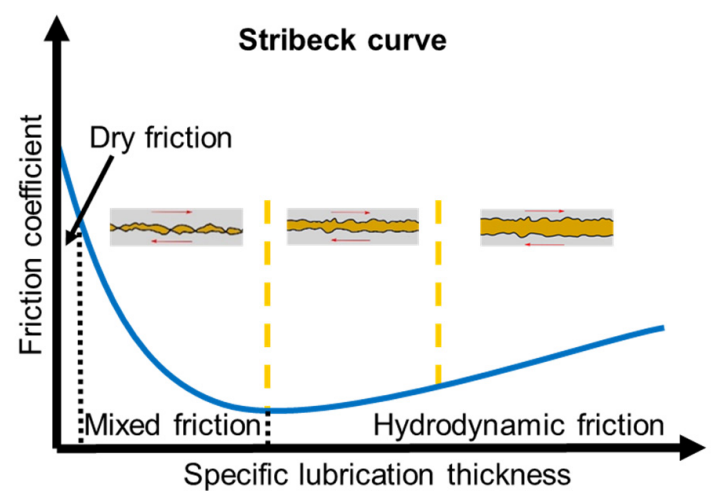

Figure 8. 3 different lubrication conditions on Stribeck curve

In the range of dry and mixed friction, peaks of roughness touch each other between the balls and the raceway. The electric analogous model of the rolling contact condition consists of an ohmic resistance caused by metallic contact, Figure 9. With a sufficient lubrication thickness, the lubricant isolates the surfaces of the ball and the raceway. A capacitor is the electric analogous model of this rolling contact condition describing the behaviour. The plate type capacitor exists between the hertzian area of the ball and the raceway. A reactance is parallely connected (Baly, 2005). With an isolation by the lubrication, the thickness prevents a bypass due to an electric arc, the rolling contact acts as a capacitator with a high reactance. The lubrication isolates the surfaces but the ratio of voltage $\mathrm{U}$ and lubrication thickness $\mathrm{H}$ is lower as the dielectric strength E (Gemeinder, 2016).

$$
E=\frac{U}{H}
$$

For thinner lubrication films, electric arcs occur and bypass the capacitator. For the electric analogous model, an EDM-switch locates beside the resistance, which closes the circuit when there is an Electric Discharge Machining (EDM) currents occur an EDM-switch locates beside the resistance closes the circuit. Due to the random behaviour of the EDM currents, the prediction of the electric behaviour is impossible. Figure 9 shows the different electric models for the possible rolling lubrication conditions. Voltage value of impedance analysers is below $1 \mathrm{~V}$, the electric model with the random switch occurs at a lubrication thickness which is below the roughness level with metallic contact and ohmic behaviour. Only the metallic contact and the isolating lubrication is important, when working with an impedance analyser (Gemeinder, 2016).

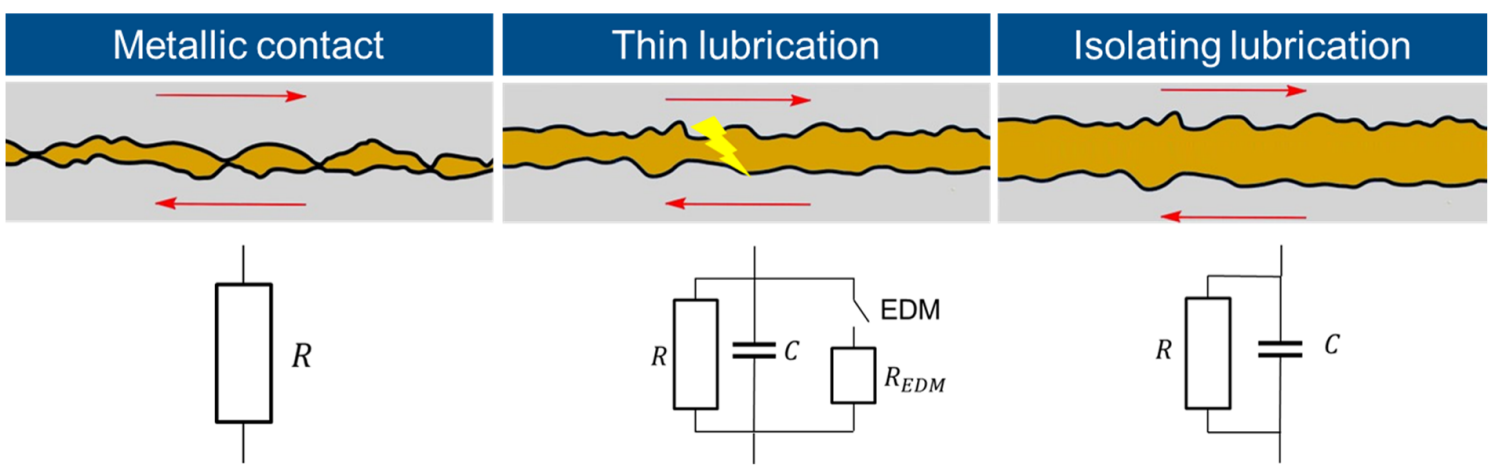

Figure 9. Electric analogous model of rolling conditions depending on lubrication thickness

Depending on the operating conditions of a ball bearing, there are five lubrication conditions based on the different conditions of a rolling contact. The operating conditions rotating speed, temperature and bearing forces influence the lubrication thickness (Hamrock and Dowson, 1981). Relative speed (ball to raceway) and temperature are different at the inner ring and the outer ring. Bearing forces are the same at inner and outer ring. The system of a ball bearing has a constant rotational frequency, but the 
outer ring has a bigger diameter than the inner ring. Additional the heat dissipation of the outer ring is higher and therefore a lower temperature of the lubricant is achieved. This leads to a thicker lubrication film between the balls and the outer ring and the outer ring achieves the isolation with lower rotational speeds, as shown in Figure 10 (Radnai et al., 2015).
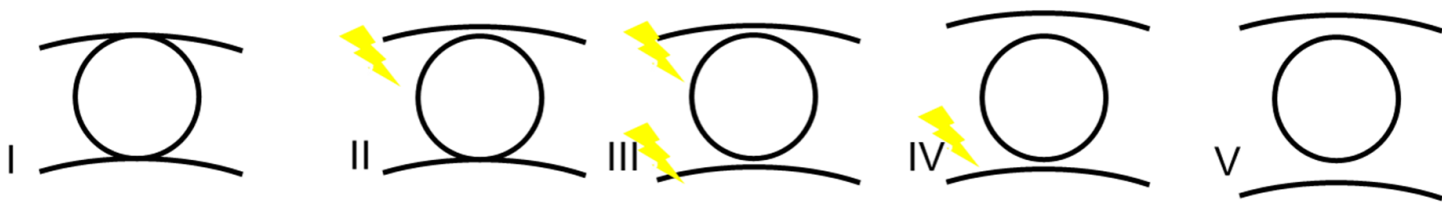

Rotation speed

Figure 10. Lubrication condition of a ball bearing depending on rotation speed

A plate capacitor describes the capacity of a rolling contact. Around the hertzian area, which function as plates of the capacitor, is an influence area that additionally acts as a capacitator (Bader et al., 2017). This area has its border at the point where the distance between ball and raceway is bigger than the lubrication thickness by the power of hundred. Figure 11 shows the capacity of a rolling contact considering two parallel capacitors, one plate type capacitor $\mathrm{C}_{\mathrm{Hz}}$ between the hertzian areas. The other one is a capacitor, which represents the area with additional influence on the capacity of a rolling contact $\mathrm{C}_{\text {air }}$ (Gemeinder, 2016).
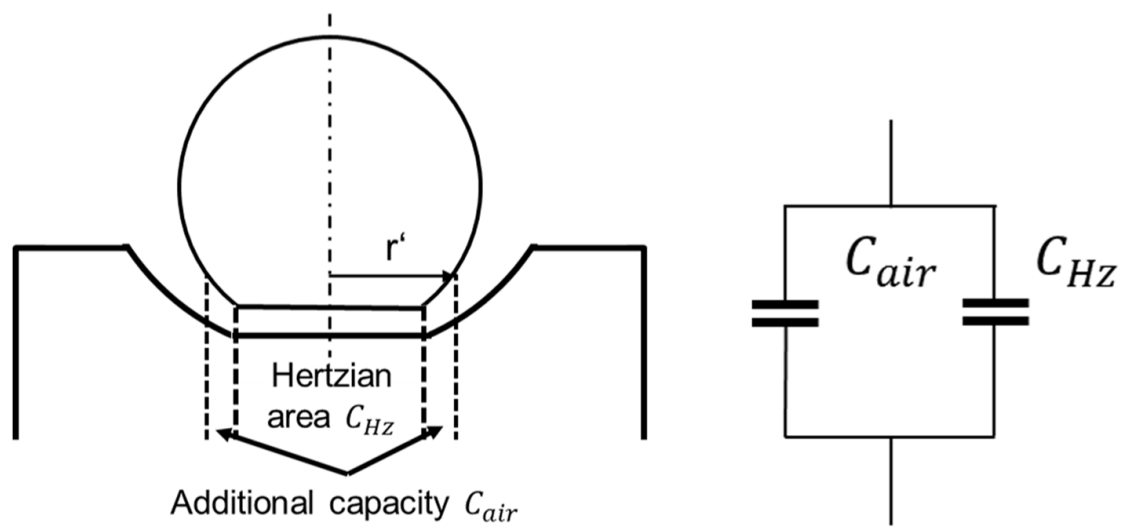

Figure 11. Hertzian area at a rolling contact and electric analogous model

The equation for the capacity of the hertzian area $\mathrm{C}_{\mathrm{Hz}}$ apllies to a plate capacitor (Gemeinder, 2016). It depends on the vacuum permittivity $\varepsilon_{0}$, the relative permittivity of the lubricant $\varepsilon_{\mathrm{r}}$, the semi major a and semi minor $\mathrm{b}$ axis of the contact ellipse of the hertzian area and the lubrication thickness $\mathrm{H}$.

$$
C_{H z}=\varepsilon_{0} \cdot \varepsilon_{r} \cdot \frac{\pi \cdot a \cdot b}{H}
$$

Because of the influence of the parallel capacity $\mathrm{C}_{\text {air }}$ the total capacity has to be corrected by the factor $\mathrm{k}_{\mathrm{c}}=1,1$ (Gemeinder, 2016).

$$
C_{r c}=k_{c} \cdot \varepsilon_{0} \cdot \varepsilon_{r} \cdot \frac{\pi \cdot a \cdot b}{H}
$$

The reactance $R_{r c}$ of the rolling contact is equal to the assumption for the estimated capacity. The lubricant's reactance between the hertzian areas depends on the semi major a and semi minor b axis of the contact ellipse, the lubrication thickness $\mathrm{H}$, the specific resistance of the lubricant $\rho$ and correction factor $\mathrm{k}_{\mathrm{r}}$ (Gemeinder, 2016).

$$
R_{r c}=k_{r} \cdot \frac{\rho \cdot H}{\pi \cdot a \cdot b}
$$


The impedance of a rolling contact $Z_{r c}$ is equal to: (Gemeinder, 2016)

$$
Z_{r c}=\frac{R_{K} \cdot \frac{1}{j \omega \cdot C_{K}}}{R_{K}+\frac{1}{j \omega \cdot C_{K}}}
$$

The impedance at one ball of the bearing $Z_{\mathrm{B}}$ is the summation of the rolling contacts impedance at the inner ring and the outer ring (Gemeinder, 2016).

$$
Z_{B}=Z_{r c i}+Z_{r c a}
$$

Figure 12 shows the inner and outer ring of the bearing connecting the balls in parallel. According to the electric analogous model, the impedance $Z_{\mathrm{BB}}$ describes a ball bearing with $\mathrm{z}$ balls (Gemeinder, 2016).

$$
\frac{1}{Z_{B B}}=\sum_{n=1}^{Z} \frac{1}{Z_{B}}
$$

In comparison to the impedance of the rolling contacts, the resistance of the bearing ring and balls is insignificant. Therefore, the impedance of the bearing only depends on the impedance of the rolling contacts.

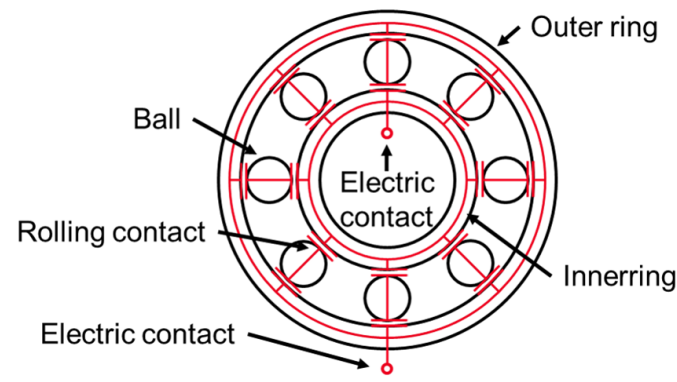

Figure 12. Electric model of a ball bearing

\subsection{Calculation of lubrication thickness}

The model of the bearing impedance presents the connection to lubrication thickness and the calculation shows the dependency to bearing load. Elastohydrodynamic lubrication of ball bearings mainly depends on three operating conditions: the operating temperature, the rotating speed and the bearing load. Additionally it depends on geometry and material parameters, which are constant in a single bearing type. Lubrication thickness decreases with increasing temperature. The viscosity increases with the temperature and high viscosity means low carrying capacity of the lubricant. Increasing rotation speed leads to an increased supply of lubricant in the gap, the result is increasing lubrication thickness. Bearing load has the contrary effect. Lubricant squeezes out of the gap and the lubrication thickness decreases. A dimensionless calculation method of Hamrock and Dawson (1981) describes the lubrication thickness depending on the influencing factors above. The dimensionless parameters are speed parameter $U$, material Parameter G, load parameter $\mathrm{W}$, the ratio $\mathrm{k}$ of the semi axis of the hertzian ellipse and the effective radius in $\mathrm{x}$ direction $\mathrm{R}_{\mathrm{x}}$.

$$
H=2,69 \cdot U^{0,67} \cdot G^{0,53} \cdot W^{-0,067}\left(1-0,61 \cdot e^{-0,73 \cdot k}\right) \cdot R_{x}
$$

The dimensionless speed parameter $U$ depends on the lubricant viscosity $\mu$, the relative speed $u$ between ball and raceway, the effective elastic modulus $\mathrm{E}^{\prime}$ and the effective radius in $\mathrm{x}$ direction $\mathrm{R}_{\mathrm{x}}$. The effective elastic modulus depends on the elastic modulus and poisson's constant of raceway and ball material. The effective radius in $\mathrm{x}$ direction depends on different radii of the ball bearing and is a constant geometry parameter. The dimensionless speed parameter depends on rotation speed and temperature (Hamrock and Dowson, 1981).

$$
U=\frac{\mu \cdot u}{E^{\prime} \cdot R_{x}}
$$


The dimensionless material parameter depends on the effective elastic modulus and the pressureviscosity-coefficient $\alpha$ of lubrication. The coefficient and therefore the dimensionless material parameter only depends on temperature (Hamrock and Dowson, 1981).

$$
G=\alpha \cdot E^{\prime}
$$

The dimensionless load parameter depends on the normal load of the calculated ball Q, the effective elastic modulus and the effective radius in $\mathrm{x}$ direction. The normal load of the ball depends linear to the radial bearing load (Hamrock and Dowson, 1981).

$$
W=\frac{Q}{E^{\prime} \cdot \mathrm{R}_{x}^{2}}
$$

The ellipticity parameter $\mathrm{k}$ is the ratio of semi major and semi minor axis of the hertzian ellipse at the raceway and the ball. The ratio depends on geometry parameters and is constant in a ball bearing (Hamrock and Dowson, 1981).

A ball bearing with the operating conditions constant temperature and rotation speed has a lubrication thickness, which only depends on the dimensionless load parameter and constant parameter c (Hamrock and Dowson, 1981).

$$
H=c \cdot W^{-0,067}
$$

If the bearing only experiences radial loads, the dimensionless load parameter depends on the radial load and constant parameters. Therefore, the lubrication thickness has a correlation to the radial load $\mathrm{F}$ (Hamrock and Dowson, 1981).

$$
H=\mathrm{c} \cdot F^{-0,067}
$$

\subsection{Calculation of the semi major and minor axis of the hertzian ellipse}

The model of the bearing impedance presents the connection to the semi axis of the hertzian area. Calculating the semi axis leads to the dependency of impedance and load. Semi major and minor axis of the hertzian ellipse mainly depends on the bearing load, material and geometry parameters (Harris, 1967). The material parameter is the effective elastic modulus. Geometry parameters are the ratio of the semi axis of the hertzian ellipse, the curvature sum $\mathrm{R}_{S}$, and the parameter $\mathfrak{C}$, which is a part of the iterative calculation to determinate the ratio of the semi axis.

$$
\begin{aligned}
& b=\left(\frac{6 \cdot \mathfrak{c} \cdot Q \cdot R_{S}}{\pi \cdot k \cdot E^{\prime}}\right)^{1 / 3} \\
& a=k \cdot b
\end{aligned}
$$

All the geometry parameters depend on radii and angels of the ball bearing. The semi axis depends on constant parameter $\mathrm{c}$ and the normal load $\mathrm{Q}$ of the calculated ball. If there is only radial bearing load, the load of a ball depends linear on the radial bearing load. Therefore, the calculations of semi axis are equal to: (Harris, 1967)

$$
\begin{aligned}
& b=\mathrm{c} \cdot \mathrm{F}^{1 / 3} \\
& a=\mathrm{c} \cdot \mathrm{F}^{1 / 3}
\end{aligned}
$$

\subsection{Calculating of bearing impedance}

The results of Chapter 4.2 and 4.3 are the dependencies of the lubrication thickness and the semi axis of the hertzian area on radial bearing load. Based on these results the calculation of bearing impedance shows the dependency of bearing impedance on bearing load. This connection is the mechanism of the sensor. The first step of the calculation is describing the impedance of a rolling contact depending on the radial bearing load. The result of an intermediate step is the linear connection of rolling contact capacity to $\mathrm{F}^{0,067+2 / 3}$ bearing load and the inverse connection of rolling contact reactance to $\mathrm{F}^{0,067+2 / 3}$ bearing load. 


$$
\begin{aligned}
& C_{r c}=k_{c} \cdot \varepsilon_{0} \cdot \varepsilon_{r} \cdot \frac{\pi \cdot a \cdot b}{H}=c \cdot \frac{F^{2 / 3}}{F^{-0,067}}=c \cdot F^{0,067+2 / 3} \\
& R_{r c}=k_{r} \cdot \frac{\rho \cdot H}{\pi \cdot a \cdot b}=c \cdot \frac{F^{-0,067}}{F^{2 / 3}}=\frac{c}{F^{0,067+2 / 3}}
\end{aligned}
$$

With the reactance and the capacity of a rolling contact, there is the calculation of the impedance of the rolling contact. The first intermediate result is the inverse connection of rolling contact impedance and $\mathrm{F}^{0,067+2 / 3}$ bearing load.

$$
Z_{r c}=\frac{R_{r c} \cdot \frac{1}{j \omega \cdot C_{r c}}}{R_{r c}+\frac{1}{j \omega \cdot C_{r c}}}=c \cdot \frac{\frac{1}{F^{0,067+2 / 3}} \cdot \frac{1}{j \omega \cdot F^{0,067+2 / 3}}}{\frac{1}{F^{0,067+2 / 3}}+\frac{1}{j \omega \cdot F^{0,067+2 / 3}}}=\frac{c}{F^{0,067+2 / 3} \cdot(j \omega+1)}
$$

The impedance of a bearing's ball is the sum of inner and outer impedance of rolling contact. Therefore, the impedance of a ball is also inverse to $\mathrm{F}^{0,067+2 / 3}$ bearing load.

$$
Z_{B}=Z_{K i}+Z_{K a}=\frac{\mathrm{c}}{F^{0,067+2 / 3} \cdot(j \omega+1)}+\frac{\mathrm{c}}{F^{0,067+2 / 3} \cdot(j \omega+1)}=\frac{\mathrm{c}}{F^{0,067+2 / 3} \cdot(j \omega+1)}
$$

The impedance of the ball bearing is a calculation of bearing ball impedance connected in parallel. Thus the bearing impedance is inverse connected to $\mathrm{F}^{0,067+2 / 3}$ bearing load.

$$
\frac{1}{Z_{B B}}=\sum_{n=1}^{z} \frac{1}{Z_{B}}=\sum_{n=1}^{z} \frac{F^{0,067+2 / 3} \cdot(j \omega+1)}{c}
$$

In case of a bearing with radial load, constant frequency $\omega$ of the analyser signal, constant temperature, and rotation speed the calculation of bearing impedance shows the inverse connection to $\mathrm{F}^{0,067+2 / 3}$ bearing load. Figure 13 shows the correlation between impedance and radial load of a bearing type 6205 with 25 ${ }^{\circ} \mathrm{C}$ operating temperature, 1500 rounds per minute and $10 \mathrm{kHz}$ frequency of the analyser signal. Especially in the load range up to $6 \mathrm{kN}$ the sensor properties are suitable to implement a precise load measuring. There are two basic challenges, the accuracy of the model and the required robustness with regard to uncertainty of tolerances and lubricant properties. When model accuracy turns out to be inefficient, measures need to be identified to enhance the predictive capability. Tolerances can be included in the analysis by detailed geometric measurements of the contact partners especially rollers, lubricant properties need to be measured on a regular basis by using test signals. Based on these considerations, the next logical steps ought to be physical testing.

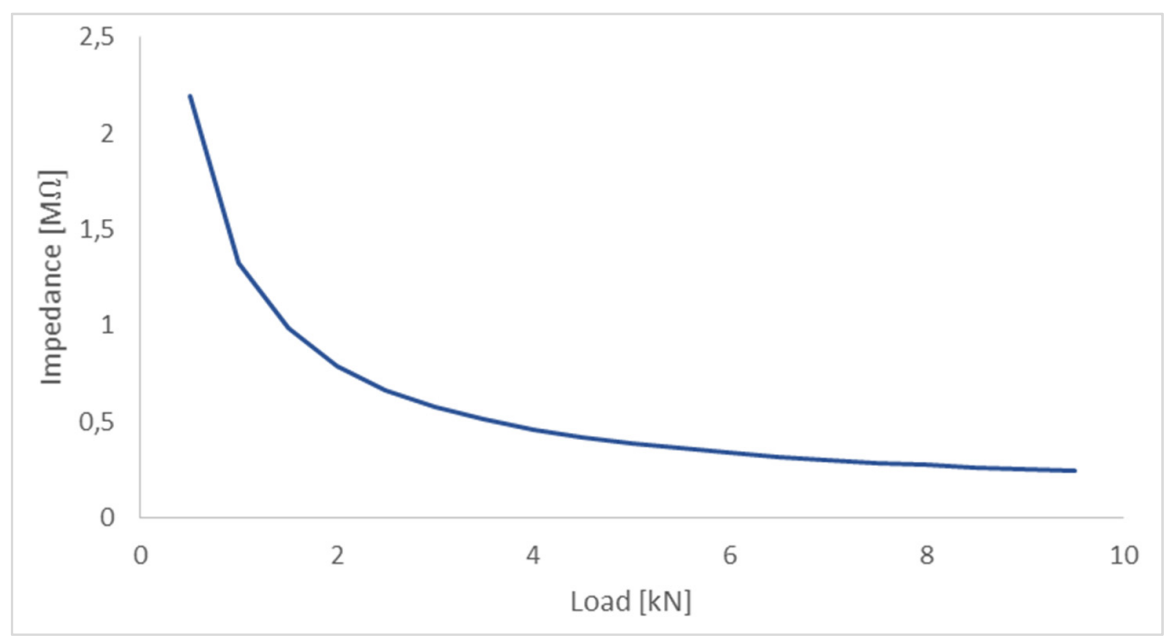

Figure 13. Correlation between load and impedance of a bearing type 6205 $\left(25^{\circ} \mathrm{C} ; 1500\right.$ 1/min; $\left.10 \mathrm{kHz}\right)$ 


\section{Conclusion}

The application of simultaneous load and failure monitoring improves the knowledge of load condition and capacity. The reduction of uncertainty in load assumption improves the estimation of components RUL. Decreasing the uncertainty in load conditions leads to only an influence of uncertainty in load capacity to the RUL estimation. Detecting an incipient crack by parallel failure monitoring reduces the uncertainty in load capacity of the component with the incipient crack and can improve its RUL estimation. The comparison of the RUL of all component offers the possibility to adjust the load distribution on the components to achieve more similar RUL of all components. The best-case result of the optimisation is the same remaining lifetime of all components, which is longer than the remaining lifetime of the component with the incipient crack without optimised operating conditions. The advantages of the method are an individual estimated and extended maintenance rate. In addition, the utilisation of components is higher before replacement. Suitable sensors, which are detecting load and failure data, are basic requirements for a successful application of the method. An example is the presented sensor mechanism for ball bearings. The mechanism consists of the dependency of bearing impedance and radial bearing load. Lubricated ball bearings form a plate type capacitor of the hertzian area at ball and raceway and the lubrication thickness as plate distance. With the influence of the radial bearing load to the semi axis of the hertzian area and the lubrication thickness, the calculation of the bearings impedance leads to an inverse correlation of the bearing impedance and $\mathrm{F}^{0,067+2 / 3}$ radial bearing load. The potentials of ball bearings are:

- Ball bearings are widespread

- Few modifications develop ball bearings to sensor ball bearings

- Bearing load is interesting monitoring data

\section{Outlook}

Following investigations quantify the advantages of the combination of load and failure monitoring to clean load or failure monitoring. Testing of the advanced sensor mechanism if it is suitable for the usage in simultaneous load and failure monitoring, follows up. After the proof of concept, there are next steps of investigations with the sensor. During the investigations, the focus is the suitability of the sensor mechanism for static and dynamic measurement. Another point is the implementation of temperature and speed measurement to use the sensor with alternating speed and temperature. The design of the sensor for the usage at operating conditions is another research area. The isolation between the bearings to the environment are design areas of special interest. Another important aspect is the connection of the analyser to the inner and the outer ring especially regarding relative speed. The difference of resistive, capacitive and inductive connection and their influence to the analyser is a point of interest. The goal is the development of a sensor for easy practical application.

\section{References}

Bader, N., Furtmann, A., Tischmacher, H. and Poll, G. (2017), "Capacitances and Lubricant Film Thicknesses of Grease and Oil Lubricated Bearings", Proceedings of the 72th Annual Meeting and Exhibition of the Society of Tribologists and Lubrication Engineers (STLE 2017), Atlanta, Georgia, May 21-25, 2017, Society of Tribologists and Lubrication Engineers (STLE), Park Ridge, Illinois, pp. 311-314.

Baly, H. (2005), Reibung fettgeschmierter Wälzlager, PhD Thesis, Leibnitz Universität Hannover.

Foulard, S. (2015), Online and real-time load monitoring for remaining service life prediction of automotive transmissions: damage level estimation of transmission components based on a torque acquisition, $\mathrm{PhD}$ thesis, Technische Universität Darmstadt.

Gemeinder, Y. (2016), Lagerimpedanz und Lagerschädigung bei Stromdurchgang in umrichtergespeisten elektrischen Maschinen, PhD thesis, Technische Universität Darmstadt.

Hamrock, B.J. and Dowson, D. (1981), Ball bearing lubrication: The elastohydrodynamics of elliptical contacts, Wiley Interscience, New York.

Harris, J.H. (1967), The lubrication of rolling bearings, Shell-Mex \& B.P., London.

Martin, G., Shork, S., Vogel, S. and Kirchner, E. (2018), "MME - Potentiale durch mechatronische Maschinenelemente", Konstruktion, Vol. 70 No. 1-2, pp. 71-75.

Mikat, H. (2015), Hybride Fehlerprognose zur Unterstützung prädiktiver Instandhaltungskonzepte in der Luftfahrt, $\mathrm{PhD}$ thesis, Technische Universität Darmstadt. 
Prashad, H. (2006), Tribology in electrical environments, Elsevier, Amsterdam.

Radnai, B., Gemeinder, Y., Kiekbusch, T., Sauer, B. and Binder, A. (2015), Schädlicher Stromdurchgang Abschlussbericht zum FVA-Vorhaben 650/I, Vol. 1127, Forschungsvereinigung Antriebstechnik e.V. (FVA), Frankfurt.

Würtenberger, J. (2017), Ein Beitrag zur Identifikation und Beherrschung von Unsicherheit bei der Erstellung von Produktmodellen, PhD thesis, Technische Universität Darmstadt.

Tobias Schirra, Master of Science

Technische Universität Darmstadt, Institute Product Development and Machine Elements

Otto-Berndt-Straße 2, 64287 Darmstadt, Germany

Email: schirra@pmd.tu-darmstadt.de 